\title{
Über den Acetylgehalt des Lignins.
}

Von

Hans Pringsheim und Hans Magnus.

(Der Redaktion zugegangen am 4. April 1919.)

Bekanntlich entsteht bei der trockenen Destillation des Holzes unter anderem ein gewisser Prozentsatz an Essigsäure. Das Holz setzt sich hauptsächlich aus drei organischen Bestandteilen zusammen: aus der Zellulose, den Pentosanen und den - Inkrustationssubstanzen, die man kurzweg als Lignin bezeichnet. Schon öfters ist die Frage erörtert worden, aus welchem der drei Holzbestandteile die Essigsäure beim Verkohlungsprozeß hauptsächlich gebildet wird; der Beantwortung dieser Frage haben ganz kürzlich Heuser und Skiöldebrand') näherzukommen gesucht, indem sie das nach der Methode von Willstätter und Zechmeister ${ }^{2}$ ) aus Holz mit starker, $42 \%$ iger Salzsäure gewonnene Lignin der trockenen Destillation unterwarfen. Sie gewannen bei der trockenen Destillation des Lignins aus Fichtenholz etwa $1 \%$ Essigsäure, wobei die Frage offen gelassen wurde, ob es sich bei dem nach der Willstätterschen Methode aus $\mathrm{Holz}$ dargestellten Lignin tatsächlich um ein unverändertes und dem natürlichen Lignin entsprechendes Produkt gehandelt hat.

In letzter Zeit und besonders unter dem Einflusse der Futterknappheit während des Krieges hat man sich bemüht, rohfaserhaltige Naturprodukte, wie Holz und Stroh, durch die Behandlung mit Alkalien, vornehmlich mit Natronlauge, verdaulich $\mathrm{zu}$ machen. Hieraus ist eine ausgedehnte Industrie entstanden, so daß es naturgemäß notwendig wurde, auch die Vorgänge zu studieren, welche bei der Einwirkung von Natron--

1) Zeitschr. für angewandte Chemie, Bd. 32, S. 41 (1919).

2) Berichte der Deutsch. Chem. Ges., Bd. 46, S. 2401 (1913).

Hoppe-Seyler's Zeitschrift t. physiol. Chemie. CV. 
lauge auf diese Materialien stattinden. Besonders erstrebeuswert erschien es natürlich, den Verbrauch an Natronlange möglichst zu beschränken und deshalb Ermittlungen darüber anzustellen, wodurch die Natronlauge bei dem sogenannten Aufschließungsprozeß hauptsächlich neutralisiert wird. Es hat sich hierbei gezeigt, daß die beim Behandeln von Holz oder Stroh entstehende Säure vornehmlich Essigsäure ist, der nur eine sehr geringe Menge Ameisensäure beigemengt war; aus diesem Grunde wurde in der Folge die gebildete organische Säure stets auf Essigsäure umgerechnet.

Schon seit längerer Zeit haben wir uns mit den Forgängen bei dem sogenannten Aufschließungsprozeß eingehend beschäftigt und hierüber Ausführliches der Öffentlichkeit übergeben ${ }^{1}$ ). Wir haben dort schon behauptet, daB mit Wahrscheinlichkeit die beim Behandeln von Holz oder Stroh mit Natronlauge gebildeten organischen Säuren zum größten Teil der Ligninsubstanz entstammen. Beim Behandeln von $\mathrm{Holz}$ oder Stroh mit Natronlauge wird die Ligninsubstanz durch Überführung in eine Ligninsäure löslich gemacht, gleichzeitig aber findet eine Verseifung der im Lignin enthaltenen Acetylgruppen statt, deren Grad von der Konzentration der Natronlauge, der Temperatur und der Dauer der Einwirkung abhängt, und die schließlich, besonders wenn man höhere Drucke als Atmosphärendruck und konzentrierte Natronlauge anwendet, eine vollkommene werden kann. Gleicbzeitig mit dieser Verseifung verändert sich die Farbe der Ligninsubstanz, und sie wird um so dunkler, je stärker der Verseifungsgrad ist. So zeigt z. B. ein mit kalter Natronlauge aus Stroh ausgezogenes und mit verdünnter Schwefelsäure gefälltes Lignin noch eine relativ helle graue bis graubraune Farbe, während ein völlig verseiftes Lignin schwarzbraun aussieht. In diesem Zusammenhange war an sich schon die dunkle Farbe des nach Willstätter gewonnenen lignins auffällig.

Die Behauptung, daß die beim Behandeln von Stroh und Holz mit Natronlauge gebildete Essigsäure zum größten Teil

1) Hans Magnus, Theorie und Praxis der Strohaufschließang. Paul Parey, Berlin 1919. 
aus dem Lignin entstammte, gründete sich des weiteren auf die Beobachtung, daß ihre Mengen ganz proportional den in diesen Materialien vorhandenen Mengen Lignin waren. Es schien uns jedoch lohnend, die Beobachtung näher zu begründen, da trotz dieser Proportionalität immerhin die Möglichkeit bestand, daß aus den anderen Bestandteilen des Holzes und Strohes ebenfalls Essigsäure, und zwar im gleichen.Verhältnis wie aus dem Lignin, gebildet würde. Aus diesem Grunde haben wir die drei hauptsächlichsten Bestandteile der Holzsubstanz bezüglich ihres Essigsäurebildungsvermögens getrennt untersucht; wir konnten jedoch natürliches Lignin für diese Versuche nicht anwenden, da solches frei von anderen Substanzen in der Natur nicht vorkommt. So waren wir gezwungen, uns des nach dor Methode von Willstätter hergestellten zu bedienen. Zuerst wurde dieses auf sein essigsäurebildendes Vermögen geprüft: hierbei stellte es sich nun heraus, daß sellost bei der energischsten Form der Einwirkung der Natronlauge unter Druck keine quantitativ nachweisbaren Mengen Essigsäure mehr aus dem Lignin gebildet wurden. Dagegen konnte dieses künstliche Lignin durch Acetylierung wieder in sein helleres Acetylprodukt zurückverwandelt werden. Wurde dieses Acetylprodukt nach Willstätter behandelt, so wandelte es sich wieder in seine schwarzbraune Modifikation um und enthielt keine Acetylgruppen mehr.

Zur Acetylierung ver'wandten wir die Methode vonVerley; $\left.{ }^{1}\right)$ das in einem Gemisch von Pyridin und Essigsäureanhydrid aufgeschwämmte Lignin geht nach und nach in Lösung und fällt beim Eingießen in Wasser in Gestalt seines Acetylproduktes wieder aus. Hier könnte natürlich der Einwand erhoben werden, daß bei diesem Acetylierungsprozeß mehr Acetylgruppen in den Ligninrest hineingebracht werden, als im ursprünglichen Holzlignin tatsächlich vorhanden waren. Daß das jedoch nicht der Fall ist, geht aus folgenden Daten hervor:

Holz (Nadelholzsägespäne) wurde mit der achtfachen Menge einer $3,5 \%$ igen Natronlauge $(30 \%$ Ätznatron vom Holzgewicht) 6 Stunden bei 6 Atmosphären behandelt. Aus

3) Vgl. L. Hochfelder, Diss. Kfünchen 1915. 
der mit Schwefelsäure angesäuerten Lösung wurde die Essigsäure mit Dampf abgetrieben und hierbei 4,2\% Essigsäure vom Holzgewicht erhalten. Die Wiederholung dieses Verfahrens orgab nach 3stündiger Behandlung noch 1,5\%, während bei dem dritten Versuch nur noch minimale Mengen Essigsäure gewonnen wurden. Die Gesamtausbeute an Essigsäure war also in diesem Falle 5,7\%. Bei einem zweiten Versuch wurde das Holz mit derselben Menge Natronlauge erst 5 Stunden am Rückflußkühler gekocht und dann mit neuer Natronlauge 1 Stunde bei 4 Atmosphären im Autoklaven behandelt. Bei der Destillation sind 6,0\% Essigsäure vom Holzgewicht erhalten worden. Das aus demselben Holz gewonnene Lignin nach Willstätter ergab bei. der Acetylierung ein Acetylprodukt, welches bei der kompletten Verseifung im ersten Versuch 19,5, im zweiten Versuch 20,2, im Mittel also 19,85\% Essigsäure zu gewinnen gestattete. Da aber im angewandten Holz nur $30 \%$ Lignin enthalten waren, so würde sich auf das Holz berechnet $\frac{19,48 \times 30}{100}=5,8 \%$ Essigsäure in guter Übereinstimmung mit unseren gefundenen Werten errechnen.

Zwecks weiteren Beweises wurde ein Versuch mit einer anderen Holzart gemacht, und zwar mit Weißbuchenholz, das einen Ligningehalt von nur $23 \%$ hat. Bei der Verseifung dieses Holzes erhielten wir im ersten Versuch 11,2, im zweiten 9,8, im Mittel also 10,5\% Essigsäure. Da wir entsprechend dem Acetylgehalt des Nadelholzlignins nur 4,8\% Essigsäure erwarten konnten, mußte es sich hier entweder um ein Lignin von einem höheren Acetylierungsgrade oder um eine Beteiligung der anderen Holzbestandteile an der Essigsäurebildung handeln. Der Acetylierungsprozeß des aus Weißbuchenholz gewonnenen Lignins ging erheblich schwieriger und langsamer vor sich als beim Nadelholzlignin: bei der Acetylierung war es nötig, von Anfang an auf dem Wasserbade zu erwärmen und den Acetylierungsprozeß länger als beim Nadelholzlignin auszudehnen. Nach der Verseifung des Acetylproduktes aus Weißbuchenholz wurden beim ersten Versuch 37,8, beim zweiten 37,9 , im Mittel also $37,85 \%$ Essigsäure gewonnen. Da in dem 
Weißbuchenholz nur $23 \%$ Lignin enthalten waren, so hätten sich $\frac{23 \times 37,85}{100}=8,7 \%$ statt der gefundenen 10,5\% Essigsäure errechnet.

Wir sehen also, daß beim Weißbuchenholz der praktisch gefundene Wert vom theoretischen doch eine außerhalb der methodischen Fehlerquelle fallende Abweichung zeigt. Eins steht unter allen Umständen fest, daß es sich in Weißbuchenholz um ein anderes Lignin als im Nadelholz handelt: daß das erstere Gruppen enthalten muß; welche die Aufnahme einer größeren Zahl von Essigsäureresten ermöglichen. Besonders bemerkenswert ist die Beobachtung, daß das Weißbuchenlignin mit einem Gehalt von $37,8 \%$ etwa doppelt soviel Acetyl als das Nadelholzlignin mit 19,85\% enthält. Das Strohlignin dagegen ist, was seinen Gehalt an Acetylgruppen betrifft, ganz ähnlich dem Nadelholzlignin zusammengesetzt. Wir erhielten bei unseren Acetylierungsversuchen des Willstätter-Strohlignins nach der Verseifung des Acetylproduktes in einem Versuche 27,4\%, in einem zweiten Versuche 27,0\% Essigsäure, bezogen auf aschefreie Trockensubstanz. Beim Acetylieren eines Strohlignins, das aus einer Strohkochungsablauge durch Ansäuern gewonnen war, erhielten wir in einem Falle 25,6\%, im zweiten Falle 26,9\% Essigsäure. Wir ersehen hieraus, daß die in den Ablaugen gelöste Ligninsubstanz sich dem Acetylierungsprozeß gegenüber genau so verhält wie Willstätter-Lignin.

Bei unseren bisherigen Versuchen haben wir die Voraussetzung gemacht, daß die Abspaltung der Essigsäure beim Behandeln von Holz mit Natronlauge unter Druck allein auf den Acetylgehalt des Lignins zurückzuführen sei. Da jedoch bei der trockenen Destillation zum mindesten aus der Zellulose ebenfalls Essigsäure entsteht, da ferner, wenigstens bein Weißbuchenholz, 1,8\% mehr Essigsäure entstanden war, als dieser Theorie entspricht, schien es uns notwendig, auch die anderen Bestandteile des Holzes, die Pentosane und die Zellulose, auf ihr Essigsäurebildungsvermögen beim Behandeln mit Natronlauge zu prüfen. 
Besonders notwendig schien diese Prüfung, da das Weißbuchenholz, wie alle Laubhölzer, einen wesentlich höheren, iiber doppelt so großen, Pentosangehalt als Nadelholz aufweist. Andererseits erhält man beim Behandeln von Stroh mit Natronlauge unter Druck, trotzdem das Stroh bedeutend mehr Pentosane als das Nadelholz enthält, doch eine geringere Menge Essigsäure.

Das Stroh haben wir in Analogie zu den verschiedenen Aufschließungsverfahren ${ }^{1}$ )

1. in der Kälte,

2. bei Kochtemperatur,

3. unter einem Überdruck von 4 Atmosphären

mit 8-12\% Natronlage vom Strohgewicht behandelt und hierbei in der Ablauge gefunden:
1. $3,2 \%$,
2. $3,9 \%$,
3. $4,1 \%$ Essigsäure.

Genau nach der gleichen Methode behandelten wir auch die Pentosane und die Zellulose.

$\mathrm{Zu}$ diesem Zwecke stellten wir uns reines Pentosan, in Gestalt von Xylan, aus Stroh nach der Methode von Salkowski dar ${ }^{2}$ ). Bei allen diesen drei Behandlungsarten entstand aus dem Xylan keine Essigsäure. Diese Beobachtung ist aus zwei Gründen wichtig: erstens liefert sie den Beweis, daß die Pentosane keine Essigsäurebildner bei der Behandlung mit Natronlauge sind, zweitens aber hatte sich gezeigt, daß3 das aus den Ablaugen gefällte Lignin ${ }^{1}$ ) je nach der Art der Behandlung mit Natronlauge nicht nur einen verschiedenen Verseifungsgrad bezüglich seines Essigsäuregehaltes aufweist, sondern da $\beta$ es auch eine gewisse Menge, bei der Destillation mit Salzsäure, Furfurol liefernde Bestandteile, also noch Pentosane enthielt. Auch hierdurch unterscheidet sich die derart gewonnene Ligninsubstanz vom Willstätter-Lignin. Da nun die Pentosane bei der Behandlung mit Natronlauge keine Esșigsäurebildung zeigen, so kann nicht der Einwand gemacht werden, daß die aus den Ablaugen ausgefällten, noch Pentoschließung.

1) Vgl. Hans Magnus, Theorie und Praxis der Strohauf-

2) Diese Zeitschr. Bd. 34, S. 166 (1901); Bd. 355, S. 340 (1902). 
sane enthaltenden Lignine einen Teil ihrer Essigsäurebildung den ihnen anhaftenden Pentosanen verdanken.

Um die Untersuchung auf die Zellulose auszudehnen, stellten wir uns eine von Lignin und Pentosanen vollkommen freie Zellulose her. Wir entfernten sowohl aus 'Holz-Zellulose (Filtrierpapier) wie aus Stroh die Ligninsubstanz durch Chlorieren nach der Methode von Croß und Bevan) ${ }^{1}$ und nachher die Pentosane durch Erhitzen mit Glyzerinschwefelsäure auf $140^{\circ}$ nach der Methode von König) ${ }^{2}$. Derartig gereinigte Zellulose unterwarfen wir, wie vorher das Stroh, den verschiedenen Aufschließungsmethoden mit Natronlæuge: beim Behandeln mit Natronlauge in der Kälte wurde keine organische Säure gebildet, beim Kochen mit Natronlauge und beim Behandeln mit Natronlauge unter Druck bei 4 Atmosphären entstanden auch aus der ganz reinen Zellulose, sowohl aus der Holz- wie aus der Strohzellulose gewisse Mengen Essigsäure, die wir in nachfolgender Tabelle zusammengestellt haben.

\begin{tabular}{|c|c|c|c|}
\hline & \multirow{2}{*}{$\begin{array}{l}\mathrm{Holzzellulose} \\
\text { mit } 40 \% \mathrm{NaOH}\end{array}$} & \multicolumn{2}{|c|}{ Strohzellulose } \\
\hline & & $\left.\operatorname{mit} 4 \% \mathrm{NaOH}^{3}\right)$ & mit $8 \% \mathrm{NaOH}$ \\
\hline 1. offen gekocht & $3,05 \%$ & $0,7 \%$ & $2,4 \%$ \\
\hline 2. unter Druck gekocht. & $4,6 \%$ & $1,7 \%$ & $3,8 \%$ \\
\hline
\end{tabular}

Das ergibt berechnet auf

\begin{tabular}{|c|c|c|c|}
\hline \multirow[b]{2}{*}{ 1. offen gekociht } & \multirow{2}{*}{$\begin{array}{c}\text { Holz } \\
\begin{array}{c}\text { (Zellulosegehalt } \\
\text { im Duchschnitt } \\
=50 \% \text { ) }\end{array} \\
1,5 \%\end{array}$} & \multicolumn{2}{|c|}{$\begin{array}{c}\text { Stroh } \\
\text { (Zellulosegehalt im Durchschnitt } \\
=40 \%)\end{array}$} \\
\hline & & $0,28 \%$ & $0,94 \%$ \\
\hline 2. unter Druck gekocht & $2,3 \%$ & $0,68 \%$ & $1,52 \%$ \\
\hline
\end{tabular}

1) CroB und Bevan, Researches on Cellulose, S. 95.

7) König, Untersuchung der landw. u. gewerbl. wichtigen Stoffe, \%. Anfl., S. 249 n. 1057 (1906).

5) Diese Konzentration dürfte den Verhältnissen beim Strolıufschln $B$ entsprechen, dà beim Aufschluß die Bälfte der angewandten $8 \%$ $\mathrm{NaOH}$ schon nach 15 Minuten verbraucht sind. 
186 Tringsheim u. Magnus, Über den Acetylgehalt des Lignins.

Annähernd dieselben Werte wie Zellulose ergab auch Glukose. Wir sehen also, daß die Zellulose auch beim Behandeln mit Natronlauge ebenso wie bei der trockenen Destillation, wonn auch in geringerem Maße, ein Essigsüurebildner ist. $\mathrm{Da}$ nun aber beim Behandeln mit Natronlauge von Stroh in der Kälte weder aus den Pentosanen noch aus der Zellulose überhaupt Essigsäure gebildet wird, so ist wenigsters in diesem Falle der Beweis ein vollkommener, daß die 3,2\% Essigsäure, die mit kalter Natronlauge aus dem Stroh gewonnen werden, ausschließlich der Ligninsubstanz entstammen. Beim•Behandeln von Holz oder Stroh mit Natronlauge, beim Kochen oder unter Überdruck kann jedoch ein geringer Teil der Essigsäure aus der Zellulose gebildet werden; auf diese Essigsäurebildung dürften die 1,8 \% Essigsäure zurückzuführen sein, welche wir beim Weißbuchenholz mehr erhalten haben, als dem Lignin dieser Holzart zukommt.

. Das Ergebnis unserer Untersuchung läßt sich folgendermaßen zusammenfassen:

1. Beim Behandeln von Holz oder Stroh mit Natronlauge in der Kälte entstammt die gesamte gebildete Essigsäure der.Ligninsubstanz. Beim Kochen von Holz oder Stroh mit Natronlauge mit oder ohne Überdruck stammt bei weitem die Hauptmenge der gebildeten Essigsäure aus dem Lignin und nur ein kleiner Anteil aus der Zellulose.

2. Das nach der Methode von Willstätter mit hochprozentiger Salzsäure gewonnene Lignin entspricht nicht mehr dem natürlichen Lignin. Es ist vollkommen verseift und enthält keine Essigsäure mehr und ist dunkel gefärbt; durch Acetylieren läßt es sich in das natürliche Lignin mit demselben Acetylgehalt und unter Rückbildung der helleren Farbe zurückverwandeln.

3. Das Lignin aus Weißbuchenholz enthält mehr, ja annähernd doppelt soviele Acetylreste als das Nadelholzlignin. 